\title{
AZIMUTHAL DEPENDENCE IN LEPTOPRODUCTION: A SIMPLE PARTON MODEL CALCULATION ${ }^{\text {th }}$
}

\author{
Robert N. CAHN \\ Department of Physics, University of Michigan, Ann Arbor, MI 48109, USA
}

Received 5 June 1978

\begin{abstract}
Semi-inclusive leptoproduction, $\ell+p \rightarrow \ell^{\prime}+h+X$, is considered in the naive parton model. The scattered parton shows an azimu thal asymmetry about the momentum transfer direction. Simple derivations for the effects in ep, $\nu$ p and $\nu p$ scattering are given. Reduction of the asymmetry due to fragmentation of partons into hadrons is estimated. The results cast doubt on the utility of such azimuthal asymmetry as a clean test of quantum chromodynamics.
\end{abstract}

Semi-inclusive leptoproduction, $\ell+p \rightarrow \ell^{\prime}+h+X$, is of special interest because, in the parton model, the observed hadron is a fragment of the struck quark parton. An extensive lore about fragmentation of quarks into hadrons has developed since the initiating work of Feynman [1] and of Bjorken and coworkers [2]. Among the motivations for studying this process were the inability of formal techniques (short distance expansions) to deal with the reaction and a proposal of Feynman that neutrino production might eventually provide a means of measuring the charge of a quark $[3,1]$.

More recently, Georgi and Politzer have proposed the process as a testing ground for quantum chromodynamics [4]. Their program is designed to show the consistency of QCD with the parton model as modified by certain $Q^{2}$ dependent, calculable corrections. It is hoped that the problem of confinement can be finessed and that the renormalization group will enable one to prove the consistency of the scheme.

As a part of their investigation, Georgi and Politzer considered the azimuthal dependence of semi-inclusive leptoproduction. If the $z$-axis is chosen to be opposite the direction of the momentum transfer, $q$, of the lepton, the observed hadron's azimuthal angle about the $z$-axis may be measured away from the $x$-axis, which is defined by choosing the $x-z$ plane to be the lepton scattering plane, with the incident and exiting leptons

\footnotetext{
Work supported in part by the Department of Energy.
}

ha ving a positive $x$-component of momentum. Most theoretical treatments of semi-inclusive leptoproduction had ignored this possible azimuthal dependence. Examining the first order perturbation theory diagrams in QCD, Georgi and Politzer found that there should be an azimuthal dependence. They suggested that this was a clean test of QCD since such effects would not arise simply as a result of the limited transverse momentum associated with confined quarks [4].

This is not so, and in fact, azimuthal effects in electroproduction were considered by Ravndal [5], and later by Kingsley [6]. Ravndal pointed out that the azimuthal dependence would be non-zero in the parton model and vanish as $1 / Q$ at fixed $x=Q^{2} / 2 M \nu$. Unfortunately, his results have not only been overlooked recently, but were stated obscurely $\neq 1$.

The proper results can be derived in a very simple way which clarifies the physical source of the asymmetry, the transverse momentum of the partons confined within the proton. After presenting this simple calculation, we shall comment on its relation to the results of Georgi and Politzer.

A convenient and appropriate approximation is to

${ }^{\$ 1}$ In particular, eq. (7) of ref. [5] indicates that averages of parton transverse momenta are required, whereas they are fixed to be the transverse momentum of the scattered (observed) partons. Also, eq. (7b) of ref. [5] must vanish as it is written. Still, the point that there are azimuthal dependences which arise from the transverse momenta of the partons was clearly stated in this paper. 
treat lepton--quark scattering as an interaction of massless particles. Then, for vector exchange (virtual photon or vector boson), the helicity of each of the fermions is conserved. Thus the scattering of electrons, say, off a proton, can be obtained as the incoherent sum of four types of scattering: left-handed electron off left-handed quark, left-handed electron off righthanded quark, etc. For charged current weak interactions, $\mathrm{V}-\mathrm{A}$ theory simply requires that only the lefthanded parts of fermions and the right-handed parts of anti-fermions be interactive.

A typical matrix element for left-left scattering is proportional to

$$
\mathscr{m}_{\mathrm{LL}}=\overline{\mathrm{u}}_{\mathrm{L}}\left(p^{\prime}\right) \gamma_{\mu} \mathrm{u}_{\mathrm{L}}(p) \overline{\mathrm{u}}_{\mathrm{L}}\left(k^{\prime}\right) \gamma^{\mu} \mathrm{u}_{\mathrm{L}}(k),
$$

where $\mathrm{u}_{\mathrm{L}}(p)=\frac{1}{2}\left(1-\gamma_{5}\right) \mathrm{u}_{\mathrm{L}}(p)$ is a spinor for a lefthanded fermion. It follows by a simple trace calculation, that

$$
\left|m_{L L}\right|^{2}=\left|m_{R R}\right|^{2}=4 s^{2},
$$

where $s=(p+k)^{2}=2 p \cdot k$. Similarly, for the scattering of a left-handed fermion by a right-handed fermion,

$\left|m_{\mathrm{LR}}\right|^{2}=\left|m_{\mathrm{RL}}\right|^{2}=4 u^{2}$,

where $u=\left(p-k^{\prime}\right)^{2}=\left(p^{\prime}-k\right)^{2}$. These matrix elements squared are the only ones we shall need.

In the lab frame, where the momentum transfer is

$q_{\mathrm{lab}}=\left(\nu, 0,0,-\sqrt{\nu^{2}+Q^{2}}\right)$,

the initial and final lepton momenta are easily found to be (just consider $q \cdot k=-Q^{2} / 2=-q \cdot k^{\prime}$ )

$k_{\mathrm{lab}}=E(1, \sin \alpha, 0, \cos \alpha)$,

$k_{\text {lab }}^{\prime}=E^{\prime}(1, \sin \beta, 0, \cos \beta)$.

The angles $\alpha$ and $\beta$ are given by

$$
\begin{aligned}
& \sin \alpha=\sqrt{\frac{Q^{2}}{\nu^{2}+Q^{2}} \frac{E^{\prime}}{E}} \cos (\theta / 2), \\
& \sin \beta=\sqrt{\frac{Q^{2}}{\nu^{2}+Q^{2}} \frac{E}{E^{\prime}}} \cos (\theta / 2),
\end{aligned}
$$

where $\theta$ is the lab scattering angle of the lepton.

In order to use the parton model, we must work in a frame in which the target hadron has high momentum. A convenient frame is the center of mass of the virtual photon and the target proton. If the proton's four-momentum is $(P, 0,0, P)$, then that of the virtual photon is $\left(q_{0}, 0,0,-P\right)$. From $P \cdot q=M \nu$ $=Q^{2} /(2 x)$, it is easy to determine that

$P=Q / 2 \sqrt{x(1-x)}$.

Thus, this center of mass frame is obtained from the lab by a boost with $\gamma \approx P / M \gg 1$. The lepton momenta in this frame are simply

$$
\begin{aligned}
& k_{\mathrm{cm}}=E(\gamma(1-\cos \alpha), \sin \alpha, 0, \gamma(\cos \alpha-1)), \\
& k_{\mathrm{cm}}^{\prime}=E^{\prime}(\gamma(1-\cos \beta), \sin \beta, \dot{0}, \gamma(\cos \beta-1)) .
\end{aligned}
$$

Now among the partons in the proton, the only ones which can be scattered by the specified virtual photon are those which carry a fraction $x$ of the proton's longitudinal momentum. (We shall assume that the parton's transverse momentum, $p_{1}$, is much less than its longitudinal momentum, $x P$.) Moreover, since the momentum transfer defines the $z$-axis, whatever transverse momentum the parton has before the scattering is its final transverse momentum. See fig. 1 . Thus the initial and final azimuthal orientation of the parton are the same. For the present we shall ignore the fragmentation of the struck quark into hadrons. That is, we shall treat the struck parton as if it were actually observed. The effects of fragmentation of partons into hadrons will be estimated afterwards.

If all the partons with longitudinal momentum fraction $x$ and transverse momentum $p_{\perp}$ (but with variable azimuthal angle $\varphi$ ) were equally likely to be scattered by the virtual photon, there would be no azimuthal asymmetry. However, this is not the case. We can represent the parton momenta by

$p=\left(x P, p_{\perp} \cos \varphi, p_{\perp} \sin \varphi, x P\right)=x P+p_{\perp}$,

where

$p_{\perp}=\left(0, p_{\perp} \cos \varphi, p_{\perp} \sin \varphi, 0\right)$.

Now the cross section for scattering of a parton is de- 

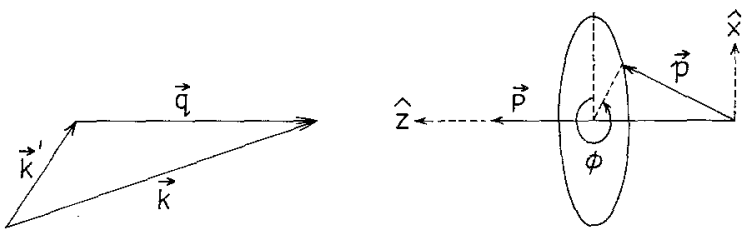

Fig. 1. Electron-parton scattering in the center of mass of the virtual photon and the target proton. The incident electron momentum, $k$, and the final electron momentum $k^{\prime}$ lie in the $x \rightarrow z$ plane. The momentum transfer $q=k-k^{\prime}$ defines the negative $z$-axis. The parton initially has momentum $p$, with $z$ component equal to a fraction, $x$, of the proton momentum, $P$. The transverse component of the parton momentum makes an azimu thal angle $\varphi$ with the $x$-axis in the $x-y$ plane. The probability of scattering depends on $\varphi$ since the invariants $s$ $=(k+p)^{2}$ and $u=\left(k^{\prime}-p\right)^{2}$ are $\varphi$ dependent. See eqs. (11) and (12). This $\varphi$ dependence is reflected in the final hadron distribution.

termined by eqs. (3) and (4). In the deep inelastic limit, $\nu \gg Q$, and $\cos (\theta / 2) \approx 1$. Using these approximations, and eqs. (5), (6) and (8), we find

$s=2 k \cdot\left(x P+P_{\perp}\right) \approx 2 x M E\left(1-\left(P_{\perp} / Q\right) \sqrt{1-y} \cos \varphi\right)$,

$-u=2 k^{\prime} \cdot\left(x P+P_{\perp}\right) \approx 2 x M E^{\prime}\left(1-\left(P_{\perp} / Q \sqrt{1-y)} \cos \varphi\right)\right.$,

where we have used the traditional variable, $y=(E$ $\left.-E^{\prime}\right) / E=q \cdot P / k \cdot P$. For electroproduction, then, we add incoherently the four helicity combinations to find the cross section (cf. eqs. (2) and (3)):

$$
\begin{aligned}
\sigma & \propto s^{2}+u^{2} \propto\left(1-\left(P_{\perp} / Q\right) \sqrt{1-y} \cos \varphi\right)^{2} \\
& +(1-y)^{2}\left(1-\left(P_{\perp} / Q \sqrt{1-y}\right) \cos \varphi\right)^{2} .
\end{aligned}
$$

The first term in eq. (12) comes from the scattering of like-helicity fermions and the second from oppositehelicity fermions. If we drop the terms in $p_{1} / Q$ we see the familiar combinations 1 and $(1-y)^{2}$ which result from the helicity structure in neutrino scattering. To lowest order in $p_{\perp} / Q$, for electroproduction,

$$
\langle\cos \varphi\rangle_{\mathrm{eP}}=-\left(\frac{2 p_{1}}{Q}\right) \frac{(2-y) \sqrt{1-y}}{1+(1-y)^{2}},
$$

$\langle\cos 2 \varphi\rangle_{\mathrm{eP}}=\left(\frac{2 p_{\perp}^{2}}{Q^{2}}\right) \frac{(1-y)}{1+(1-y)^{2}}$.

The expressions for charged current weak interactions (in the approximation in which anti-quarks in the nucleon are ignored) is obtained by restricting the interaction to left-handed quarks and can be read directly from eq. (12). Thus we find

$\sigma_{\nu} \propto\left(1-\left(2 p_{\perp} / Q\right) \sqrt{1-y} \cos \varphi\right)^{2}$,

$\sigma_{\bar{v}} \propto(1-y)^{2}\left(1-\left(2 p_{\perp} / Q \sqrt{1-y}\right) \cos \varphi\right)^{2}$.

In the valence quark approximation, then

$\langle\cos \varphi\rangle_{\nu \mathrm{p}}=-\left(2 p_{1} / Q\right)(1-y)^{1 / 2}$,

$\langle\cos \varphi\rangle_{\nu \mathrm{p}}=-\left(2 p_{1} / Q\right)(1-y)^{-1 / 2}$,

$\langle\cos 2 \varphi\rangle_{\nu \mathrm{p}}=\left(p_{\perp}^{2} / Q^{2}\right)(1-y)$,

$\langle\cos 2 \varphi\rangle_{\nu \mathrm{p}}=\left(p_{\perp}^{2} / Q^{2}\right)(1-y)^{-1}$.

We see that the quark parton model does give azimuthal dependence for the semi-inclusive cross section. In fact, we would have obtained similar results if we had used scalar partons. The analogous matrix element would have been

$m^{\text {scalar }}=\overline{\mathrm{u}}\left(k^{\prime}\right) \gamma_{\mu} \mathrm{u}(k)\left(p+p^{\prime}\right)^{\mu}$,

and its square

$\left|\mathscr{M}^{\text {scalar }}\right|^{2}=8 s(-u)$

From eq. (11) then,

$$
\begin{aligned}
& \sigma^{\text {scalar }} \propto 2(1-y)\left(1-\left(p_{\perp} / Q\right) \sqrt{1-y} \cos \varphi\right) \\
& \times\left(1-\left(p_{\perp} / Q \sqrt{1-y}\right) \cos \varphi\right)
\end{aligned}
$$

where proportionality has the same normalization as in eq. (12). The similarity between eqs. (12) and (12') demonstrates that the phenomenon of azimuthal dependence does not require that the parton have spin. Indeed, in the limit $y \rightarrow 1$, the equations are identical, which reflects the equality of the current $\bar{u}\left(p^{\prime}\right) \gamma^{\mu} \mathbf{u}(p)$ 
$=\overline{\mathrm{u}}\left(p^{\prime}\right)\left[\left(p+p^{\prime}\right)^{\mu}-\mathrm{i} q \gamma^{v \mu}\right] \mathrm{u}(p) / 2 M$ and the convective current, $\left(p+p^{\gamma}\right)^{\mu}$, in the limit $q \rightarrow 0$. Even if the electrons were treated as spinless as well, there would be an azimuthal dependence since then $m \propto(k$ $\left.+k^{\prime}\right)_{\mu}\left(p+p^{\prime}\right)^{\mu} \propto(s-u) \propto(2-y)-\left(p_{\perp} / Q\right) \sqrt{1-y}$ $X \cos \varphi$. We conclude that azimuthal dependence in vector exchange interactions is inevitable since the partons have transverse momentum as a consequence of being confined and such dependence certainly does not require any special mechanism like gluon bremsstrahlung.

The treatment above is artificial since no allowance has been made for the decay of the struck parton into observable hadrons. There are two features which can be anticipated on general grounds. First, the fastest observed hadron will not carry all of the momentum of the struck parton, but just some faction $z<1$ of it. Thus the transverse momentum of the struck parton might be guessed to be $p_{\perp} / z$, where $\boldsymbol{p}_{\perp}$ is the transverse momentum of the observed hadron. Secondly, the strength of the azimuthal asymmetry will be reduced by the random transverse momentum added by the quark decay.

These effects can be estimated by adopting a model for the transverse momentum distribution of partons in a hadron and for the transverse momentum given to hadrons in the quark decay. Suppose that both these distributions are gaussian:

$f\left(x, p_{\perp}\right) \propto \mathrm{e}^{-a p_{\perp}^{2},} \quad D\left(z, p_{\perp}\right) \propto \mathrm{e}^{-b p_{\perp}^{2}}$

where $f$ represents the quark distribution and $D$ the fragmentation function. Let the $z$-direction be defined as in fig. 1. Then the longitudinal momentum of the struck parton is $x P$ and that of the observed hadron is $z x P$. If the transverse momentum of the struck parton is $p_{1 \perp}$ and that of the observed hadron is $\boldsymbol{p}_{\perp}$, then the momentum of the observed hadron transverse to the parton direction is (for $z x P \gg\left|\boldsymbol{p}_{1 \perp}\right|,\left|\boldsymbol{p}_{1}\right|$ ) just $\boldsymbol{p}_{\perp}-z \boldsymbol{p}_{1 \perp}$. Suppose the azimuthal dependence for the parton cross section is

$\frac{\mathrm{d} \sigma}{\mathrm{d}^{3} p_{1}} \propto\left[A+B p_{1 \perp} \cdot \hat{\boldsymbol{x}}+C\left(2\left(p_{1 \perp} \cdot \hat{\boldsymbol{x}}\right)^{2}-p_{1 \perp}^{2}\right)\right] \mathrm{e}^{-a p_{1 \perp}^{2}}$

which is the general form arising from eq. (16a). Then the distribution for the observed hadrons will vary as

$$
\begin{gathered}
\mathrm{d} \sigma / \mathrm{d}^{3} p \propto \int\left\{A+B p_{1 \perp} \cdot \hat{x}+C\left[2\left(p_{1 \perp} \cdot \hat{x}\right)^{2}-p_{1 \perp}^{2}\right]\right\} \\
\times \mathrm{e}^{-a p_{1 \perp}^{2}} \mathrm{e}^{-b\left|p_{\perp}-z p_{1 \perp}\right|^{2}} \mathrm{~d}^{2} p_{1 \perp}
\end{gathered}
$$

or, carrying out the integration,

$$
\begin{aligned}
& \frac{\mathrm{d} \sigma}{\mathrm{d}^{3} p} \propto\left[A+B \frac{p_{\perp}}{z} \cdot \hat{\boldsymbol{x}}\left(\frac{b z^{2}}{a+b z^{2}}\right)\right. \\
& \left.\quad+C\left(2\left(\frac{p_{\perp} \cdot \hat{\boldsymbol{x}}}{z}\right)^{2}-\left(\frac{p_{\perp}}{z}\right)^{2}\right)\left(\frac{b z^{2}}{a+b z^{2}}\right)^{2}\right] .
\end{aligned}
$$

The strength of the azimuthal asymmetry is measured by the ratios $B / A$ and $C / A$. Eq. (19) then shows the effects we anticipated. If the decay function introduces no transverse momentum $(b \rightarrow \infty)$, the only modification of $B / A$ and $C / A$ in going from eq. (17) to eq. (19) is that the observed hadron transverse momentum should be replaced by $p_{1} / z$ before being interpreted as the transverse momentum of the parton. If the decay does introduce transverse momentum ( $b$ finite) the azimuthal asymmetry is reduced. A reasonable assumption would be $a=b$, in which case the dilution of the asymmetry is very significant: the $\cos \varphi$ term is reduced by $z^{2} /\left(1+z^{2}\right)$ and the $\cos 2 \varphi$ term by the square of that factor. Since the highest observed $z$ for a typical event is about 0.4 , this effect is very important. Similar corrections would also have to be made for the gluon brems. strahlung model of ref. [4].

While the general formulas derived in ref. [4] using QCD and perturbation theory are fairly complex, in the limit $z \rightarrow 1$ the result is

$\langle\cos \varphi\rangle_{\mathrm{ep}}=-\frac{\alpha_{\mathrm{s}}}{2} \kappa \sqrt{1-z} \frac{(2-y) \sqrt{1-y}}{1+(1-y)^{2}}$,

where $\alpha_{\mathrm{s}}$ is the $Q^{2}$-dependent strong coupling constant which may be about 0.2 at $Q^{2}=10 \mathrm{GeV}^{2}$ and which varies very slowly, as $\left(\log Q^{2} / Q_{0}^{2}\right)^{-1}$. The constant $\kappa$ is about unity. Eq. (20) may be compared to eq. (13a). The predictions are strikingly different. The parton model result at fixed $p_{\perp}$ varies as $Q^{-1}$ while the QCD result varies only slowly with $Q^{2}$.

The relation between the parton model result and the QCD result bears some resemblance to the predictions for $R=\sigma_{\mathrm{L}} / \sigma_{\mathrm{T}}$ in ordinary electroproduction. The 
parton model predicts [1] $R \approx 4\left\langle p_{\perp}^{2}\right\rangle / Q^{2}$, where $\left\langle p_{\perp}^{2}\right\rangle$ is the average transverse momentum squared of the partons at a given value of $x$. The QCD prediction for $R$ is small and slowly varying. The parton prediction for $R$ has an apparent $1 / Q^{2}$ dependence, but this could be modified if the parton distributions are $Q^{2}$ dependent so that $\left\langle p_{\perp}^{2}\right\rangle$ also depends on $Q^{2}$. In contrast, for the azimuthal asymmetry, the value of $p_{\perp}$ is fixed so that this extra $Q^{2}$ dependence would be absent.

That the QCD prediction vanishes in the limit $z \rightarrow 1$ is easy to understand: the transverse momentum arises solely from recoil against gluon emission and in the limit $z \rightarrow 1$ the exiting parton carries nearly all the momentum and the gluon none. In contrast, the parton model predicts the largest asymmetry for large $z$.

It is reasonable to expect that there will be azimuthal asymmetry coming from both the transverse momentum of the partons and from the QCD effects described in ref. [4]. Using eqs. (13a) and (20), we can compare their expected sizes. Roughly speaking, the parton transverse momentum contribution dominates the QCD piece if $Q<4 p_{\perp} / \alpha_{\mathrm{s}} \approx 20 p_{\perp}$. In reality, the predictions for both models should be diluted by substantial factors as demonstrated in eqs. (17) and (19). As a result, rather accurate, high statistics experiments with incident muons or neutrinos will be required to observe these effects.
I would like to acknowledge the advice and stimulation of my colleagues M. Einhorn, G. Kane, Y. Kazama, B. Roe, D. Sinclair, and J. Vander Velde.

\section{References}

[1] R.P. Feynman, Photon hadron interactions (Benjamin, 1972).

[2] See, e.g.: E.W. Colglazier, Hadronic final states in inclusive leptoproduction and colliding beams, i.e. features of current fragmentation, in: Proc. Summer Inst. on Particle physics (1973) Vol. 1, SLAC Rep. No. 167.

[3] R.P. Feynman, talk given at the Europhys. Conf. (Balatonfured, Hungary, 1972);

G.B. Farrar and J.L. Rosner, Phys. Rev. D7 (1973) 2747; R.N. Cahn and E.W. Colglazier, Phys. Rev. D9 (1973) 2658.

[4] H. Georgi and H.D. Politzer, Phys. Rev. Lett. 40 (1978) 3; see also: J. Cleymans, Angular correlations in lepton hadron scattering, Univ. of Bielefeld preprint, unpublished.

[5] F. Ravndal, Phys. Lett. 43B (1973) 301.

[6] R.L. Kingsley, Phys. Rev. D10 (1974) 1580; see also: T.P. Cheng and A. Zee, Phys. Rev. D6 (1972) 885 ;

A.M. Kotsinyan, Teor. Mat. Fiz. 24 (1975) 206; Engl. transl. Theor. Math. Phys. 24 (1976) 776. 